DOE/PC/88935--TII

DE92 017922

\title{
INHIBITION OF RETROGRESSIVE REACTIONS IN COAL/PETROLEUM CO-PROCESSING
}

\section{QUARTERLY TECHNICAL PROGRESS REPORT}

Reporting Period: January 1, 1992 - March 31, 1992

Harold H. Schobert

Jasna Tomic

May 1992

Contract No. DE-FG22-88PC88935

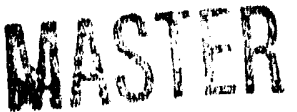




\section{TABLE OF CONTENTS}

O B JE CT IVE 1

S U M M A R Y

CHARACTERIZATION OF PETROLEUM RESIDS ..........................2

Fractionation of Petroleum Resids ......................................2

Elemental Analysis of Resid Fractions .................................. 3

Separation of Fractions by Liquid Chromatography ...................4

Nuclear Magnetic Resonance Spectroscopy of Petroleum Resid

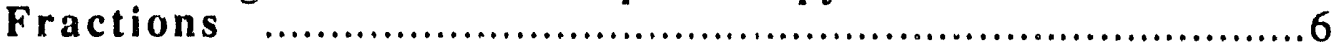

13C NUCLEAR MAGNETIC RESONACE SPECTROSCOPY OF THE

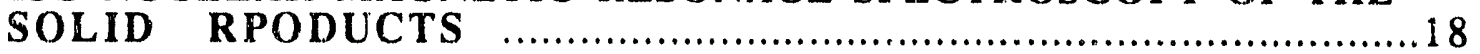

CONCLUSIONS

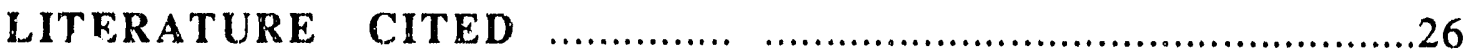

DISCLAIMER

This report was prepared as an account of work sponsored by an agency of the United States Government. Neither the United States Government nor any agency thereof, nor any of their empluyees, makes any warranty, express or implied, or assumes any legal liability or responsibility for the accuracy, completeness, or usefulness of any information, apparatus, product, or process disclosed, or represents that its use would not infringe privately owned rights. Reference herein to any specific commercial product, process, or service by trade name, trademark, manufacturer, or otherwise does not necessarily constitute or imply its endorsement, recommendation, or favoring by the United States Governisent or any agency thereof. The views and opinions of authors expressed herein do not necessarily state or reflect those of the United States Government or any agency thereof. 


\section{OBJECTIVE}

The overall objective of this project is to develop a fundamental understanding of the reactions occurring at the onset of coke formation during the co-processing of coals with petroleum residua. The specific objectives include examination of chemical components, or groups of components, in coals and petroleum feedstocks to quantify and rank the effects of these components in retarding or enhancement of coke formation. The work involves bench scale reactions in microautoclaves, supplemented by studies of the carbonaceous residues by such techniques as diffuse reflectance Fourier transform infrared spectroscopy and ${ }^{13} \mathrm{C}$ nuclear magnetic resonance spectrometry.

During this reporting period work focused on Task 4, identification of mechanisms of coke formation. The objective of this task is to identify those compounds or components which are specifically responsible for initiating coke formation. Major components of the petroleum resids were analyzed using solvent separation techniques and instrumental methods such as nuclear magnetic resonance spectrometry and gas chromatography. The analytical data obtained in this task will allow for identification of coke precursors and establish a ranking system for predicting compatibility of particular coals and petroleum resids as feedstocks for coprocessing with minimal coke formation.

\section{SUMMARY}

Characterization of the petroleum resids and their solvent separated fractions (maltenes and asphaltenes) were continued. The whole resid and the fractions were analyzed by elemental analysis. In addition, the maltene fractions were further separated using open column liquid chromatography into saturate, aromatic, and polar fractions. The saturate fractions were analyzed by gas chromatography. Nuclear magnetic spectroscopy was useu to collect spectroscopic data on the the petroleum resids and the solvent fractions. This data combined with elemental analysis is used to define the types of chemical structures typical of the project resids.

The saturate fractions of the Hondo resid were found to be 'lighter' than the saturates of Blend and West Texas resids. In terms of the saturates, the Blend and West Texas resids are very similar. The chromatographic analysis and the NMR data indicate that the West Texas resids are more aromatic. The results of coprocessing experiments have shown that West Texas resid is the best feedstock of the three used in this project. The 'good' performance of West Texas resid under coprocessing conditions is related to its chemical structure. 


\section{CHARACTERIZATION OF PETROLEUM RESIDS}

\section{Fractionation of Petroleum Resids}

The petroleum resid samples were fractionated previously into pentane-solubles (maltenes) and pentane-insolubles (asphaltenes). This separation was repeated several times to verify the results. The method of separation was described previously $[1,2]$. As noted earlier, the asphaltene content of the petroleum resids increases in the order : Hondo $<$ Blend $<$ West Texas vacuum resid. The obtained fractions were then subjected to further analysis. The pentane-soluble fractions (maltenes) were analyzed using gas chromatography (GC) and gas chromatography coupled with mass spectrometry (GC/MS). Unfortunately, only the maltene fraction from Hondo resid was amenable to this analysis, while the maltene fractions from Blend and West Texas vacuum resids were not. The maltene fractions of the Blend and West Texas vacuum resids are rich in high molecular weight compounds which will not elute under the conditions of the gas chromatographic analysis.

The maltene fractions were further separated by open column liquid chromatography into safurates (pentane-soluble), aromatics (benzene-soluble), and polars (tetrahydrofuran-solubles). This separation gives a rough estimate on the qualitative differences of the maltenes and therefore the whole resids, but also concentrates the compounds according to molecular type and weight. The three fractions are then analyzed by GC and GC/MS.

Nuclear magnetic resonance spectroscopy analysis was performed on the maltene and asphaltene fractions. The hydrogen and carbon aromaticities were measured directly from the ${ }^{1} \mathrm{H}$ and ${ }^{13} \mathrm{C}$ NMR spectra.and can be used to calculate some average structural parameters. The ratio of aromatic hydrogen to aromatic carbon was used to estimate the degree of ring substitution or ring condensation in the petroleum resid samples ar: their solvent fractions. The calculated parameter in conjunction with the hydrogen ard proton aromaticity data can be used to define the structural differences between the samples. 
Elemental Analysis of Resid Fractions

Elemental analysis was performed on the whole resid samples and their solvent fractions using a Leco $\mathrm{C}, \mathrm{H}, \mathrm{N}-600$ instrument and a Leco sulfur titrator. The results of the analysis are shown in Table 1.

Table 1. Elemental analysis of petroleum resids and resid fractions

\begin{tabular}{|c|c|c|c|c|c|}
\hline Whole: & $\% \mathrm{C}$ & $\% \mathrm{H}$ & $\% \mathrm{~N}$ & $\% S$ & $\mathrm{H} / \mathrm{C}_{\text {at }}$ \\
\hline Hondo & 76.19 & 10.17 & 0.65 & 5.2 & 1.59 \\
\hline Blend & 84.56 & 10.25 & 0.58 & 4.8 & 1.44 \\
\hline West Texas & 84.52 & 10.28 & 0.57 & 3.2 & 1.45 \\
\hline \multicolumn{6}{|l|}{ Maltenes: } \\
\hline Hondo & 82.2 & 11.34 & 0.35 & 9.7 & 1.64 \\
\hline Blend & 83.86 & 10.55 & 0.33 & 6.0 & 1.50 \\
\hline West Texas & 82.93 & 10.89 & 0.33 & 3.5 & 1.56 \\
\hline \multicolumn{6}{|l|}{ Asphaltenes: } \\
\hline Hondo & 80.34 & 8.59 & 1.88 & 7.0 & $1.27^{-}$ \\
\hline Blend & 83.56 & 7.96 & 1.19 & 6.7 & 1.13 \\
\hline West Texas & 83.33 & 7.8 & 1.30 & 6.7 & 1.11 \\
\hline
\end{tabular}

The carbon content of Hondo resids is the lowest at $76.19 \%$ while the values for Blend and West Texas resid are very close. The differences are even smaller for the maltene and asphaltene fractions. Overall, the carbon contents of the whole materials and their solvent fractions are rather uniform. Similarly, we observe that the range of values for hydrogen content is equally small. Note that the values for carbon and hydrogen of Blend resid and W. Texas resid are strikingly similar. We have seen this similarity in the NMR data of the Blend and West Texas resid spectra as well. This might be the result of similar process history of the two samples, which were both obtained from Amoco Company and were classified as vacuum resids. On the other hand, Hondo resid is much lighter and as indicated by the supplier, only $63 \mathrm{wt} \%$ is a fraction boiling above $1000^{\circ} \mathrm{F}$. This value is 91 $w t \%$ and $85 w t \%$ for West Texas and Blend resid respectively.

The greatest variations are observed in the sulfur content between the three samples. Hondo resid has the highest sulfur content consistently in the whole resid as well as in the maltene and asphaltene fractions. Perhaps it is somewhat unusual that the sulfur content of the Hondo asphaltene is lower than that of the respective maltene fraction, since one would expect a higher concentration of heteroatoms in the asphaltene fractions in general. In the case of the Blend vacuum resid and $W$. Texas vacuum resid we find that indeed a greater amount of sulfur is present in the asphaltene then in the maltene fractions. The nitrogen 
content of the whole resids and the fractions indicates that upon solvent fractionation nitrogen concentrates in the asphaltene fractions leaving the maltenes nitrogen-poor.

The last column in Table 1 compares the atomic $\mathrm{H} / \mathrm{C}$ ratios of the resid samples and their respective solvent fractions. The $\mathrm{H} / \mathrm{C}$ ratio of the maltenes is higher than that of the original whole resid and it is lower for the asphaltene fraction. This is consistent with the assumption that the more aromatic compounds will concentrate in the asphaltene fraction. Hondo resid has highest $\mathrm{H} / \mathrm{C}$ ratio while Blend resid and West Texas resid have very similar $\mathrm{H} / \mathrm{C}$ values especially for the whole sample. The maltene and asphaltene fractions have a wider distribution of $\mathrm{H} / \mathrm{C}$ ratios.

\section{Separation of Fractions by Liquid Chromatography}

The maltenes were separated into three fractions using open column liquid chromatography. The method is described in the previous report [2]. Approximately 300 mg of sample was loaded onto the column and eluted with $100 \mathrm{ml}$ of pentane, benzene, and tetrahydrofuran to obtain the saturate, aromatic and polar fractions.

Table 2. Fractionation data for resid maltenes.

\begin{tabular}{|c|c|c|c|}
\hline Maltene sample & Saturates, wt\% & Aromatics, wt\% & Polars, wt\% \\
\hline Hondo & 32.50 & 46.43 & 21.07 \\
Blend & 16.47 & 46.99 & 36.54 \\
West Texas & 25.58 & 49.15 & 25.26 \\
\hline
\end{tabular}

All three samples have very similar percentages of aromatics but differences are found in the percentage saturate and polar fractions. Hondo resid maltenes are richest in saturates among the three samples and contain approximately twice as much saturates as the Blend vacuum resid maltenes. Comparing the amount of polar compounds, the values in Table 2 indicate that the situation is reversed. Namely, the highest amount of polars is present in Blend resid maltenes and the lowest in Hondo maltenes. The distribution of the three fractions indicates the compositional and structural differences of the resid samples.

High resolution capillary column chromatography was used to characterize the separated saturate fractions. A Perkin-Elmer 8500 gas chromatograph with a fused silica capillary column ( $\mathrm{Rt}_{\mathrm{x}}-50$; crossbonded $50 \%$ methyl $-50 \%$ phenyl polysiloxane $0.25 \mathrm{~mm}$ i.d. $x 30 \mathrm{~m}$ ) was used with $\mathrm{He}$ as a carrier gas and flame ionization detector. The column was programmed from $40^{\circ} \mathrm{C}$ to $280^{\circ} \mathrm{C}$ at a heating rate of $4^{\circ} \mathrm{C} / \mathrm{min}$ and isothermal time 5 and $10 \mathrm{~min}$ respectively. The samples were dissolved in pentane and $1 \mu \mathrm{l}$ was injected at a 


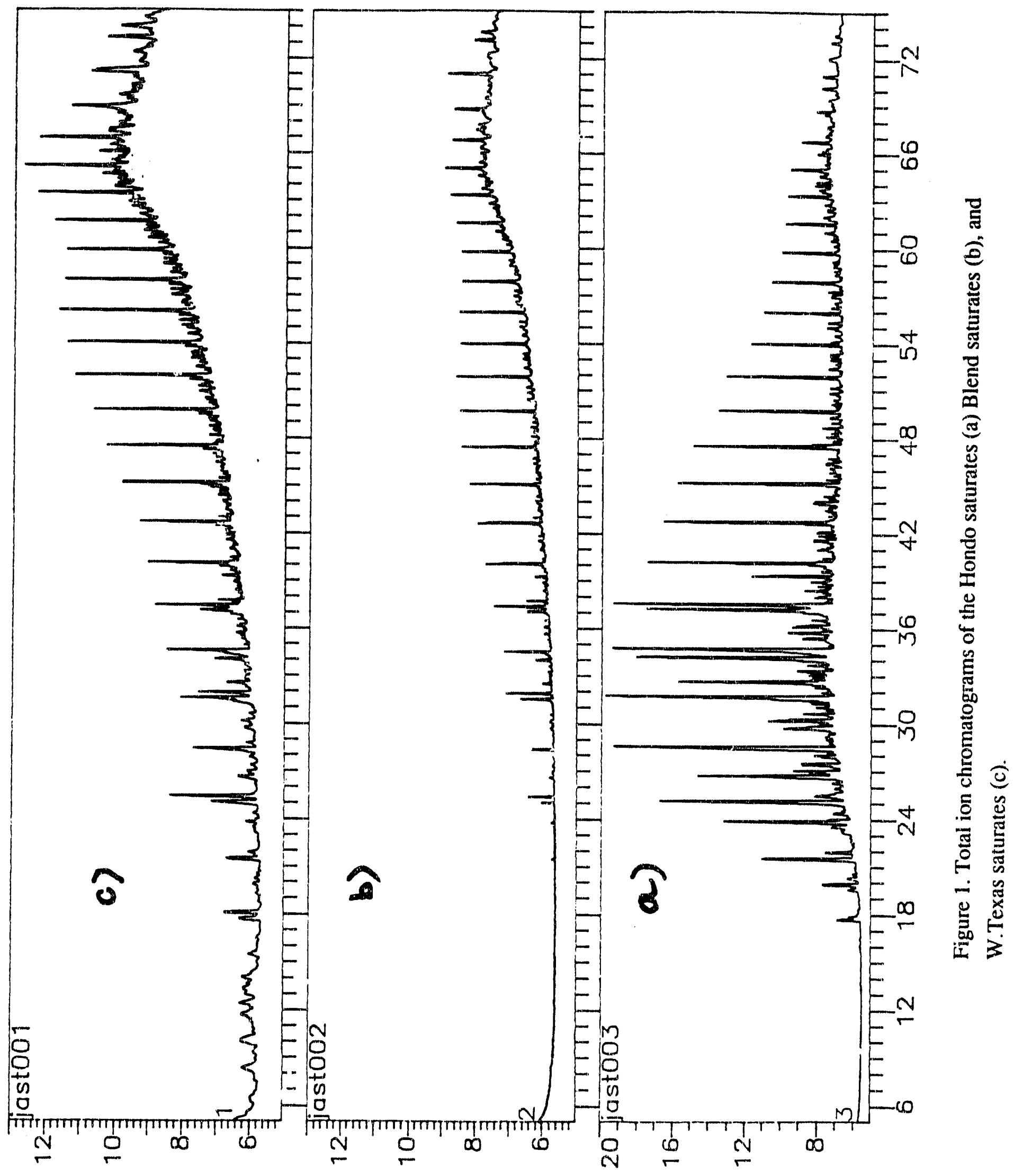


splitless mode. Figure 1 compares the total ion chromatograms of the saturate fractions of the Hondo maltenes (a), Blend maltenes (b), and W. Texas maltenes (c).

The chromatograms indicate that Hondo saturates are the lightest of the three samples and that most of the compounds elute in 75 minutes. According to the retention time and previous analysis using the identical column and temperature program, certain peaks were assigned. The typical alkane pattern is easily identified. The Hondo saturates exhibit the strongest peaks between $C_{15}$ to $C_{18}$ alkanes (retention time 28 to $38 \mathrm{~min}$ ) with the distribution maximum at $\mathrm{C}_{16}$. After 38 minutes, the peaks decrease gradually in intensity. The last identifiable peaks in the alkane series are assigned to $\mathrm{C}_{32}$ and $\mathrm{C}_{33}$. The chromatogram of the Hondo saturates contains relatively small amounts of compounds eluting at longer retention times. On the contrary, Blend and West Texas saturates are much 'heavier' samples containing relatively smaller amounts of the lower boiling alkanes as compared to the Hondo.The peak heights of the alkane series shown in Figure $1 \mathrm{~b}$ and $\mathrm{c}$ are very close, showing a relatively equal distribution of the alkane series. Noticeable on both chromatograms $b$ and $c$ are the unresolved 'humps' at higher retention times which are attributed to high molecular weight compounds.

Very close resemblance is found when the chromatogram of the saturate fraction is compared with the previously obtained chromatogram of the maltene fraction for Hondo resid sample. This indicates that the method for concentrating the saturates by open column liquid chromatography was successful. Analysis of the aromatic and polar fractions was not possible due to the much higher molecular weight of compound found in these fractions which do not elute in the GC.

\section{Nuclear Magnetic Resonance Spectroscopy of Petroleum Resid Fractions}

Nuclear magnetic resonance analysis was performed on the maltene and asphaltene fractions. The proton spectra were recorded on a Bruker WM-360 Pulse FT NMR spectrometer operating at a frequency of $360 \mathrm{MHz}$ and were reported in the previous quarterly report [2]. ${ }^{13} \mathrm{C}$ NMR spectra were recorded on the same instrument at an operating frequency of $90.5 \mathrm{MHz}$ with inverse gate proton decoupling on during acquisition time.

The major separation in the ${ }^{13} \mathrm{C}$ NMR spectra is between aromatic and aliphatic carbon, which enables direct determination of carbon aromaticity. The hydrogen aromaticity was previously determined from the ${ }^{1} \mathrm{H}$ NMR spectra. Unfortunately, this technique does not provide more detailed information when applied to resids and their solvent fractions. The resolution in the aromatic regions $\left(9.00-6.00\right.$ for ${ }^{1} \mathrm{H}$ and $148-118$ 
for ${ }^{13} \mathrm{C}$ ) is poor due to the complex mixture of aromatics present in the resids. In the aliphatic regions there is nore resolution, although the features of the spectra are very similar. The spectra of maltenes exhibit more features than those of the asphaltenes which are heavier and not completely soluble.

Comparing the ${ }^{13} \mathrm{C}$ NMR spectra of the whole resid samples and their maltene and asphaltene fractions, it is evident that the maltene spectra resemble very well the original whole resid. The features in the aliphatic regions are very similar which is expected since the solvent fractionation will lead to a concentration of the aliphatic components in the maltenes. The arnmatic region in the maltene spectra shows three peaks which were not present in the original spectra. These could be a result of residual toluene which was used to dilute the original sample before mixing with pentane. The aliphatic region in the asphaltene spectra shows fewer features than the original spectra of the whole sample. Specifically there is a loss of peaks in the region from $\sim 22-29 \mathrm{ppm}$. This region is assigned to certain carbons $B$ to aromatic ring and $\mathrm{CH}_{2}$ in ring-joining ethylene groups. The loss of peaks in this region could be interpreted as a result of loss of longer chain structures and increased conjugation of rings.

The distribution of aromatic and aliphatic carbons was determined by integrating the respective regions and normalizing over the complete spectrum. The values of the hydrogen and carbon aromaticities of the maltene and asphaltene fractions and the whole resid samples are presented in Table 3. The last column of the Table 3 represents the aromatic hydrogen to aromatic carbon ratio calculated on an atomic basis. This parameter can provide a rough estimate on the ring conjugation and/or ring substitution and essentially estimate the number of free aromatic hydrogens.

Table 3. Hydrogen and carbon aromaticities of petroleum resid fractions.

\begin{tabular}{|c|c|c|c|}
\hline Sample & $\mathrm{H}_{\mathrm{ar}}, \%^{\mathrm{a}}$ & $\mathrm{C}_{\mathrm{ar}}, \% \mathrm{~b}$ & $\left(\mathrm{H}_{\mathrm{ar}} / \frac{\left.\mathrm{C}_{\mathrm{ar}}\right)(\mathrm{H} / \mathrm{C}) \text { at }}{}\right.$ \\
\hline Hondo-maltenes & 5.33 & 20.21 & 0.433 \\
Blend-maltenes & 6.62 & 30.21 & 0.328 \\
W. Texas-maltenes & 8.51 & 36.98 & 0.360 \\
\hline Hondo-asphaltenes & 7.44 & 37.33 & 0.254 \\
Blend-asphaltenes & 12.80 & 53.08 & 0.270 \\
W. Texas-asphaltenes & 10.48 & 51.47 & 0.227 \\
\hline Hondo resid & 8.43 & 25.44 & 0.528 \\
Blend resid & 9.73 & 28.57 & 0.492 \\
W. Texas resid & 8.64 & 32.29 & 0.388 \\
\hline
\end{tabular}

$a$ from proton NMR data. $b$ from ${ }^{13} \mathrm{C}$ NMR data. 


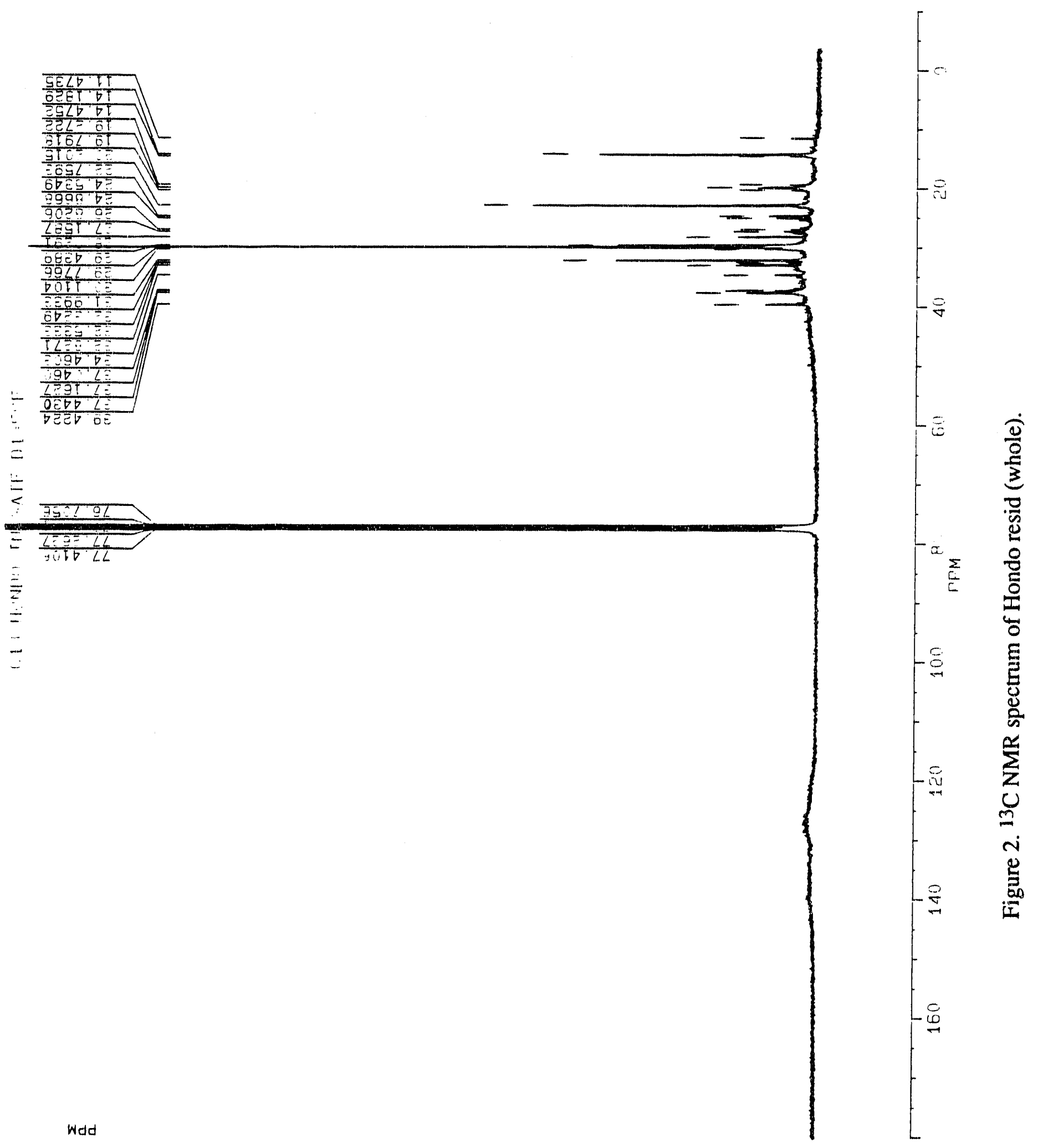




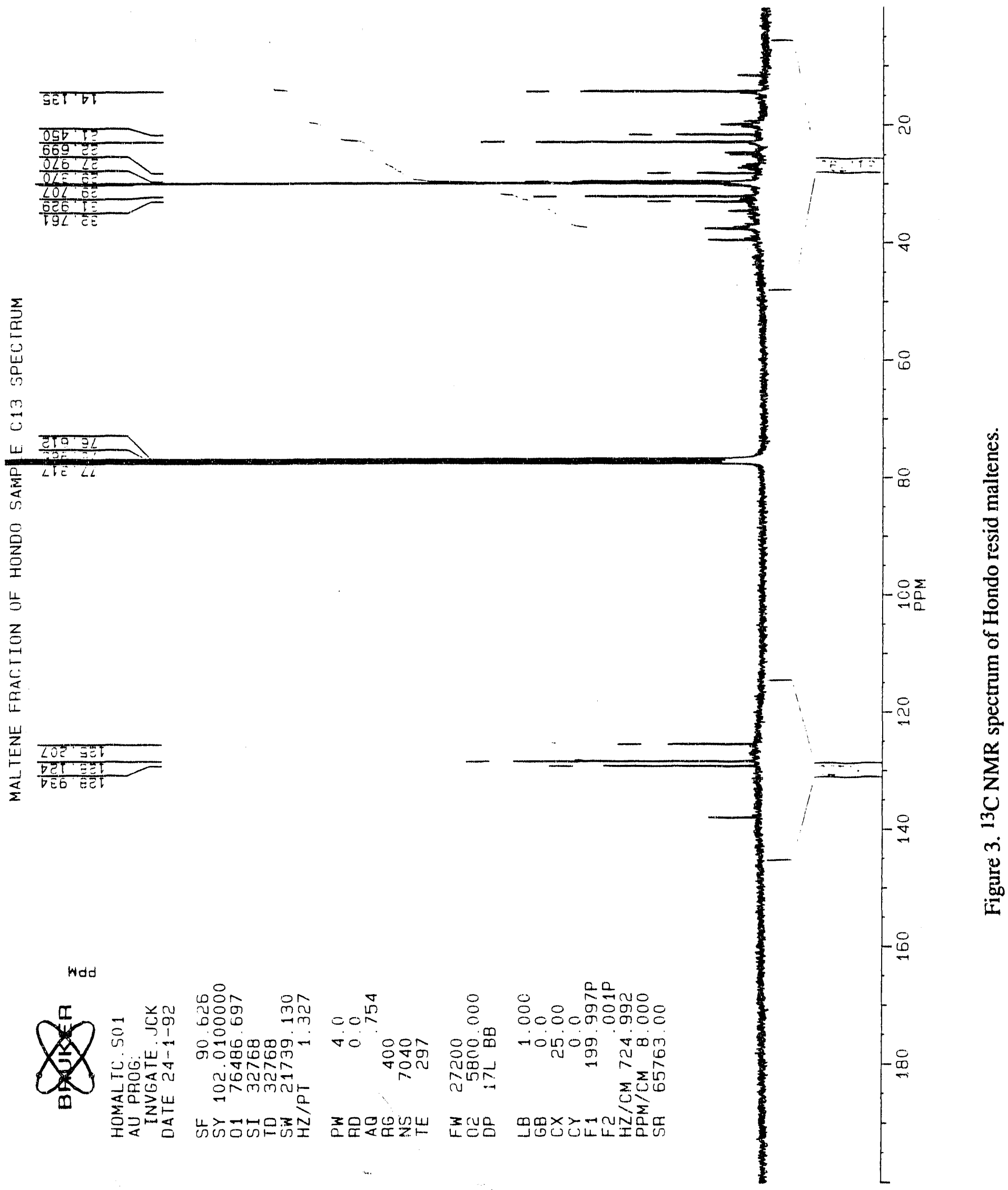




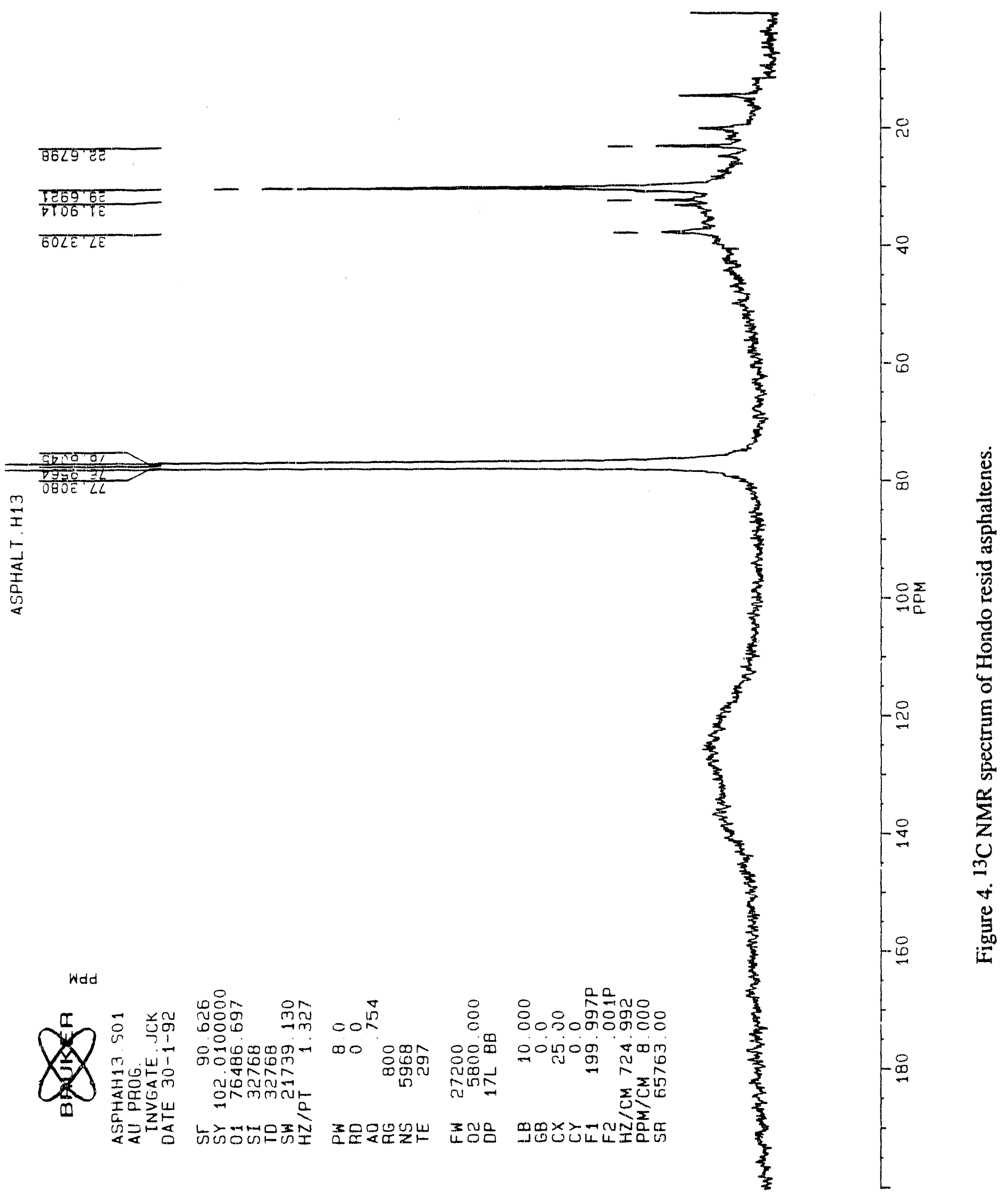




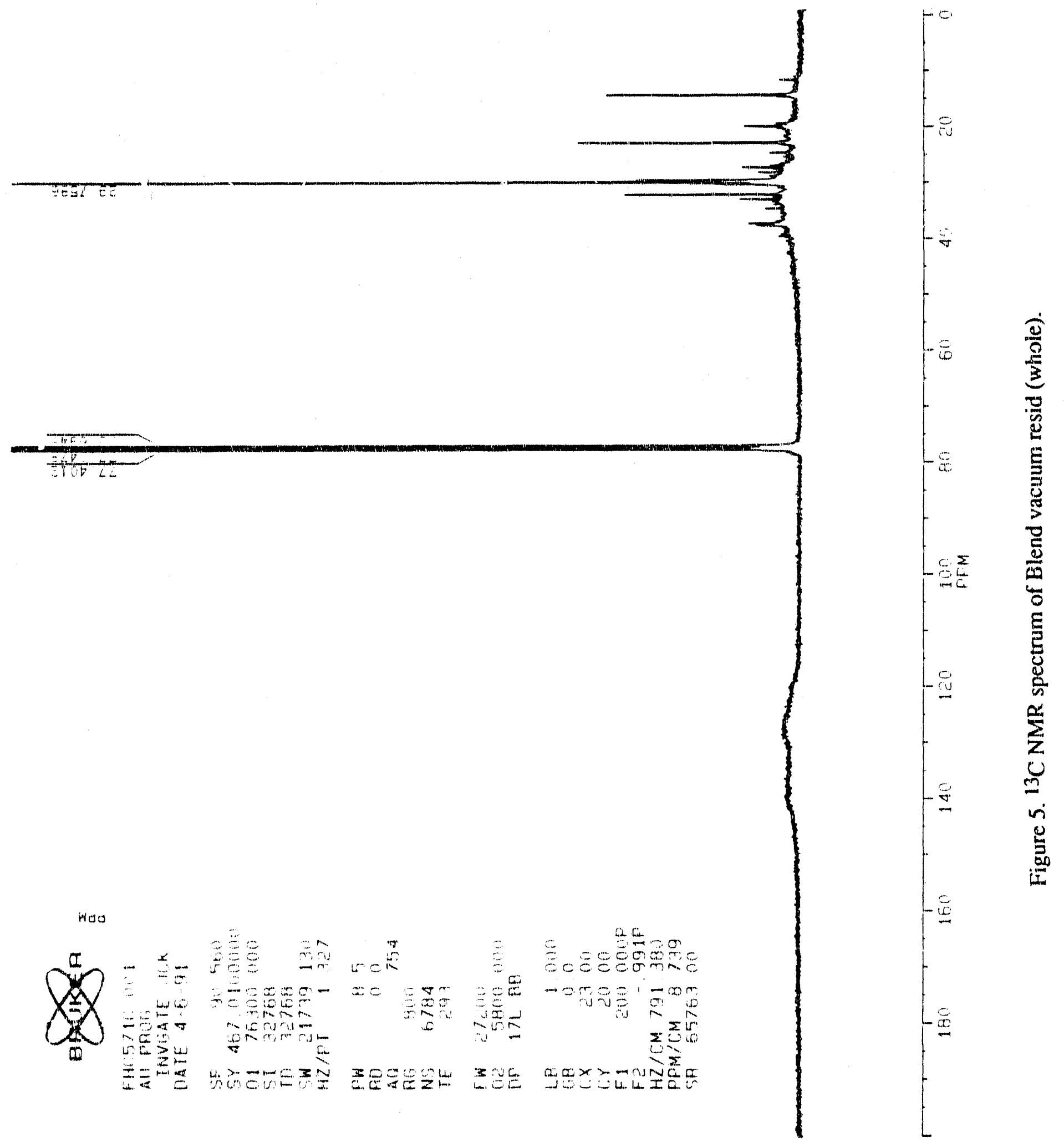




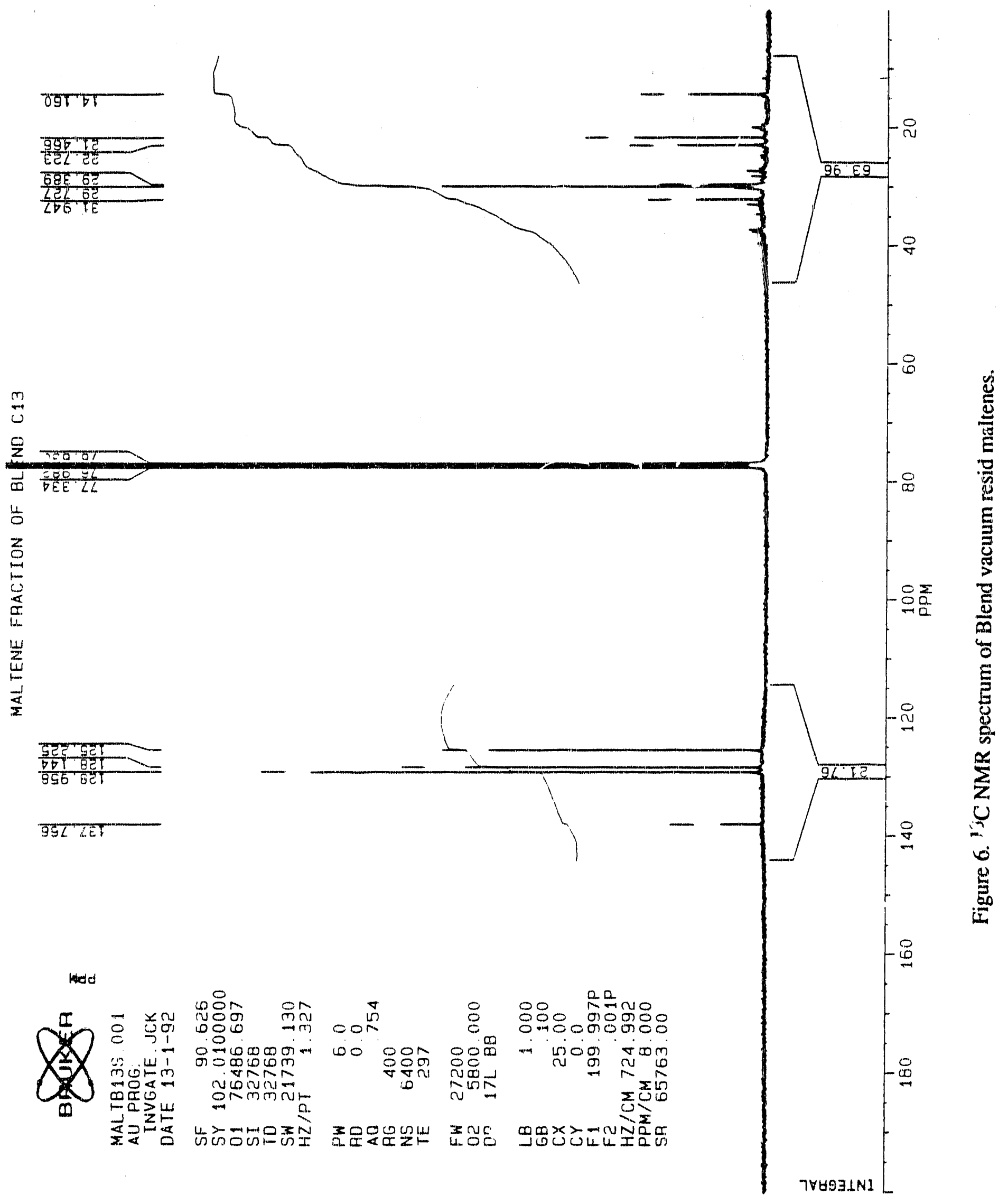




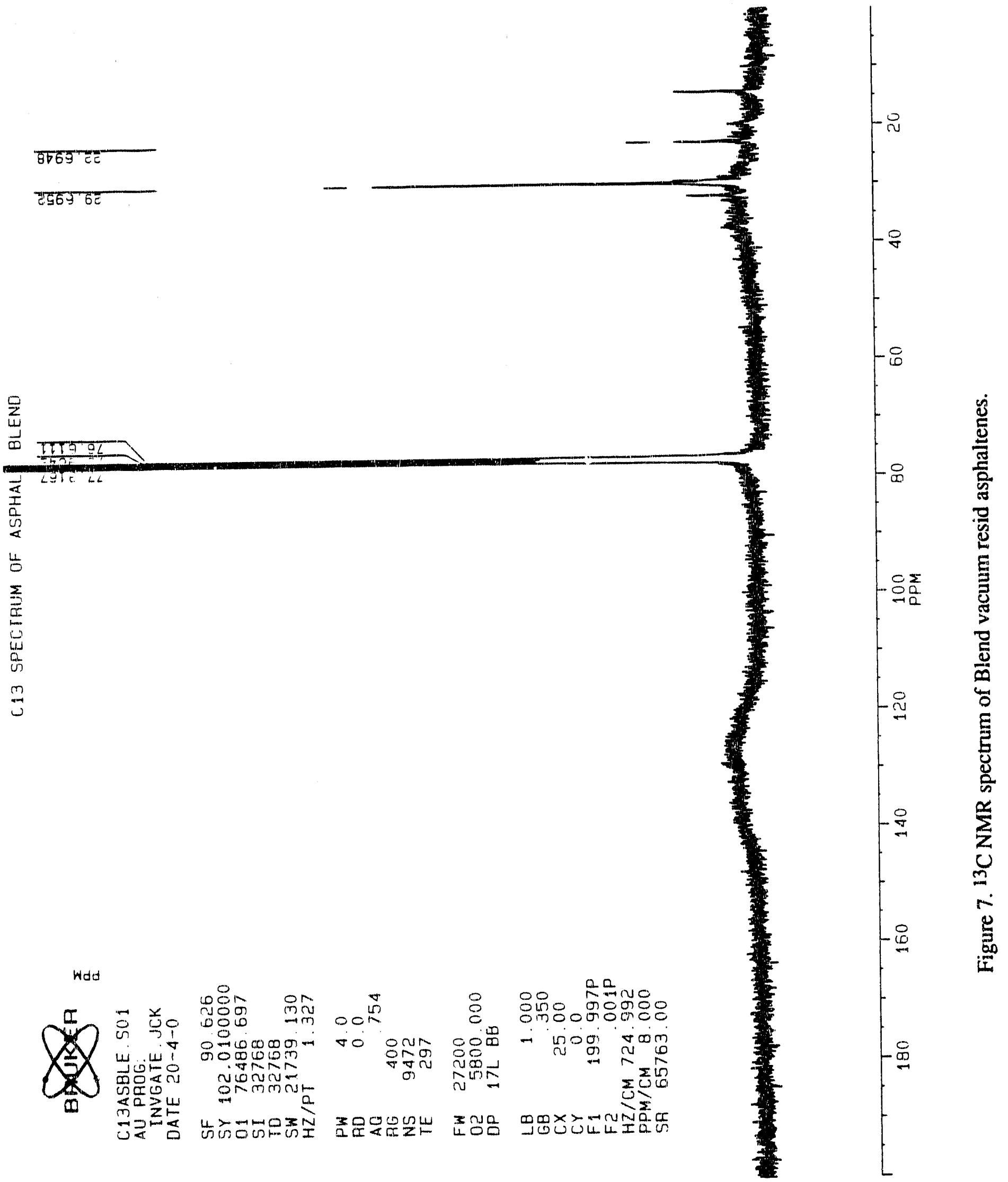




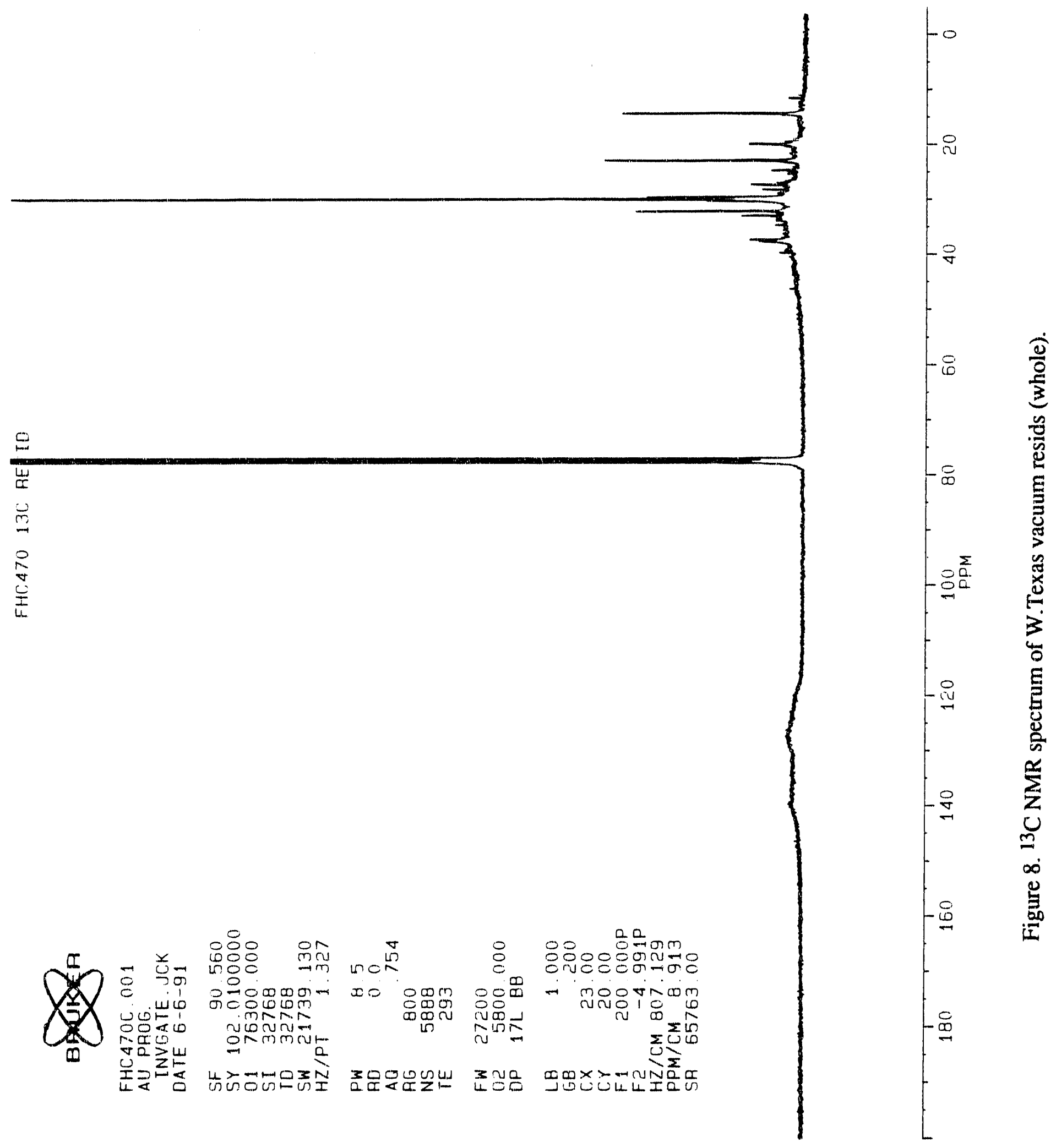




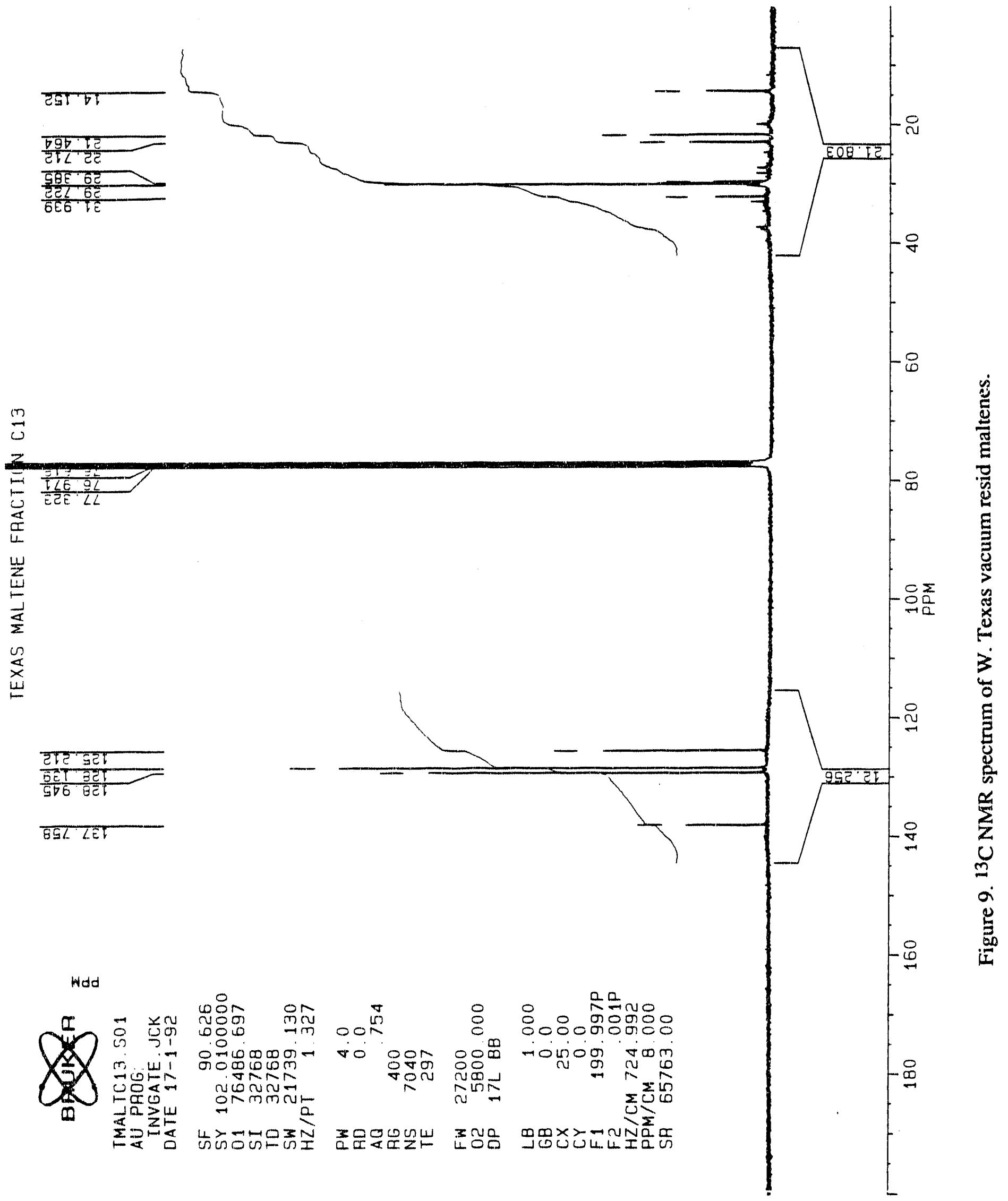




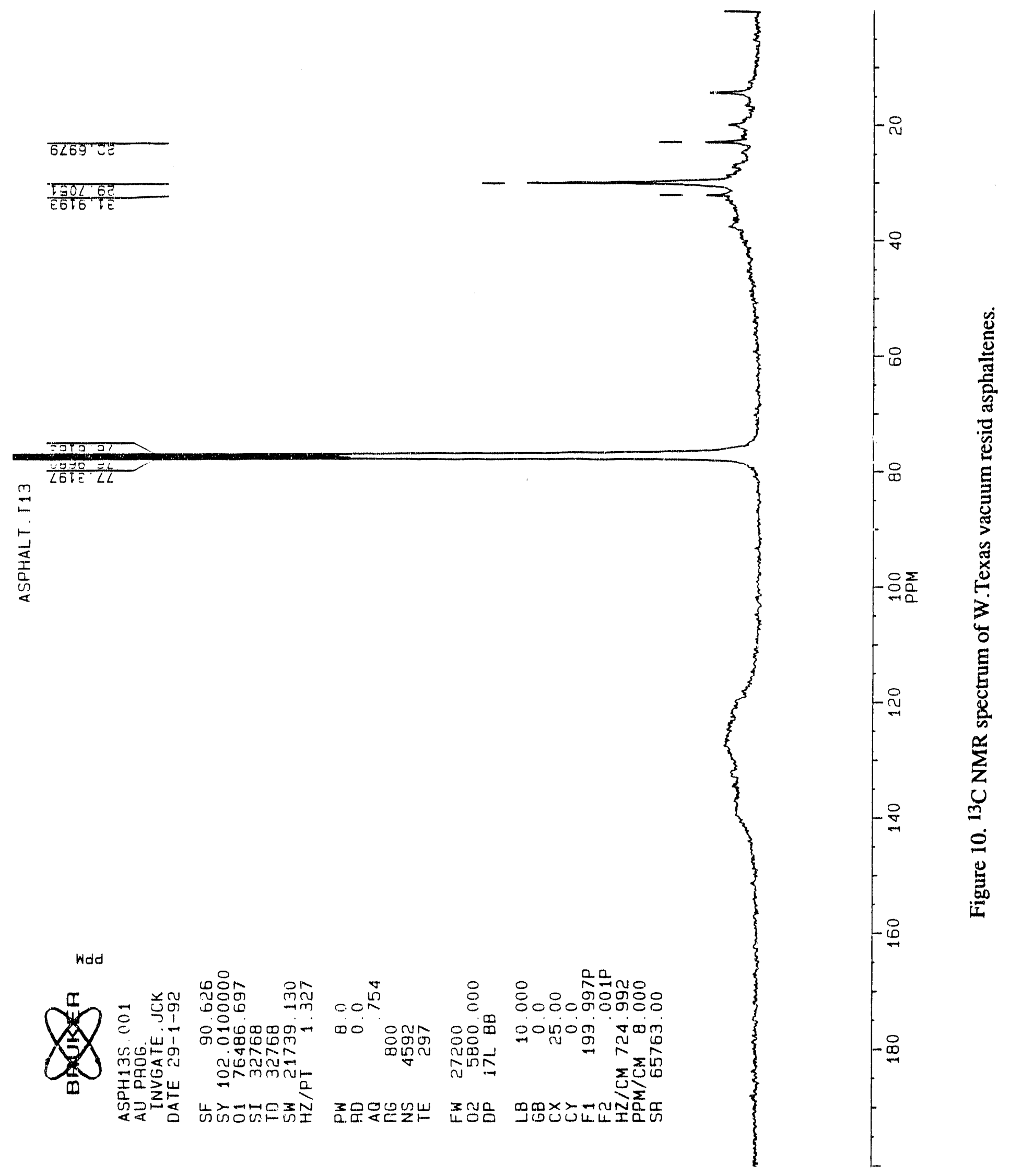


The atomic ratio of aromatic hydrogen to carbon is lowest for the asphaltenes indicating that the asphaltenes basically consist of aromatic ring systems which are highly conjugated or substituted. The lowest value amongst the asphaltenes is that of West Texas asphaltenes, which according to this parameter would consist of larger aromatic ring systems then the Blend or Hondo asphaltenes. It is interesting to note that the change in the calculated $\mathrm{H}_{\mathrm{ar}} / \mathrm{C}_{\mathrm{ar}}$ for the asphaltene and the parent original resid is greatest for Hondo resid. Table 3 shows a value of 0.53 for the whole resid and only 0.25 for the asphaltene fraction. This change is much smaller when we compare the values for the case of Blend resid and especially West Texas resid.

From the analytical results obtained on the resids and their solvent fractions some general conclusions can be dri wn. Hondo resid is definitely the lightest of the three resids. The weight percent saturates is the highest in Hondo resid and the GC of the saturates and (earlier the maltenes) shows a distribution maximum at $\mathrm{C}_{16}$. Elemental analysis data supports the highly paraffinic character of Hondo resid by the highest $\mathrm{H} / \mathrm{C}$ ratio. The other interesting piece of information provided by the elemental analysis is the percent $S$ and $N$, which distinguishes Hondo resid from the other two samples as the one which contains the most heternatoms. Blend and West Texas resid have very similar elemental composition but the differences between these two samples are structural. Liquid chromatography separation showed that Blend resid has a higher amount of polar compounds while West Texas has a higher percentage of aromatics. The amount of saturates is lower in Blend resid, but from the GC chromatograms it seem that the chemical composition of the saturates is very similar, namely, both samples contain the series of long alkanes discributed equally. The distinct feature of West Texas resid is the degree of ring substitution or ring conjugation. Even though it seems from the determined hydrogen aromaticity that Blend resids contains more protons attached to the aromatic rings, when one pairs this data with the carbon aromaticity, the resulting $\mathrm{H}_{a r} / \mathrm{C}_{\mathrm{ar}}$ indicates a significant difference in terms of the number of 'free' protons. In the light of this information it is possible to conclude that West Texas vacuum resid contains larger polyaromatic structures and short alkyl side chains, while Blend resid contains slightly less aromatics but longer alkyl side chains. Another interesting parameter to evaluate would be the amount of labile hydrogen which can be estimated by NMR of the whole sample and the saturate fraction. The labile hydrogen can be an indicator of hydrogen donation ability of the particular resid. 


\section{${ }^{13}$ C NUCLEAR MAGNETIC RESONANCE (NMR) SPECTROSCOPY OF THE SOLID PRODUCTS}

The solid THF-insoluble residues from the coprocessing experiments were analyzed by solid state ${ }^{13} \mathrm{C}$ NMR using a Chemagnetics NMR Model M100s located in the laboratories of the Fuel Science Program. The experiments were standard cross polarization/magic angle spinning (CPMAS) and pulsed Fourier transform (PFT) technique to collect the NMR spectrum. The conditions used were: contact time $1000 \mu \mathrm{s} ;$ carbon frequency $25 \mathrm{MHz}$; pulse width $6.0 \mu \mathrm{s}$; and pulse delay 1s. The acquisition count was 5000 or 10000 in some cases. The THF-insoluble solids were obtained from reactions at $450^{\circ} \mathrm{C}$ were chosen for comparison. These solids were derived from the coprocessing experiments of PSOC 1488, a Montana subbituminous coal and PSOC 1504, a Utah hVA bituminous coal with the three petroleum resids.

The collected spectra of THF-insoluble solids from coprocessing experiments conducted at $450^{\circ} \mathrm{C}$ of PSOC 1488 and PSOC 1504 with Hondo, Blend, are shown in Figures 11 to 16. The CPMAS spectra display two main broad peaks; one for aromatic carbons between 100 and $160 \mathrm{ppm}$ and the other for aliphatic carbons between 0 and 60 $\mathrm{ppm}$. The resonance at $\sim 155 \mathrm{ppm}$ arises from aromatic carbons bearing an oxygen functional substituent. The broad peaks at 275 and $-10 \mathrm{ppm}$ are from the spinning sidebands of the aromatic carbons.

All the spectra have a strong aromatic peak, while the aliphatic peaks seem to vary slightly with respect to the resid used in the experiment. The shape of the aromatic peak is different from that in the spectrum of the original unreacted coal, which was acquired and reported earlier [3]. The original coal contained two 'shoulders' on the aromatic peak; one at $142 \mathrm{ppm}$ assigned to bridgehead carbons or catechol-like structures and one at $155 \mathrm{ppm}$ most likely due to phenolic carbons [4]. These peaks are not visible in the spectra shown here, indicating a loss of the these groups from the insoluble residue. In the region around $175 \mathrm{ppm}$, a broad peak is present which is usually assigned to carbonyl carbons. The presence of this peak is more visible the spectra from PSOC 1488 coal than from PSOC 1504 coal. A curve resolving program will be used to integrate these regions and compare them quantitatively. Solids from reactions conducted at reaction temperature $400^{\circ} \mathrm{C}$ will be analyzed by the NMR technique and compared to the ones at hand. 


\section{CONCLUSIONS}

The collected data on the coprocessing reactions of the coals and the resids have shown that there are differences in the degree of coal conversion based on the resid used. We have reported earlier that coal conversions were highest at $400^{\circ} \mathrm{C}$ regardless of the coal or the resids. The composition of the resids influences the coal conversion at temperatures of $450^{\circ} \mathrm{C}$. The assorted analytical techniques performed on the petroleum resids indicate that the structural components of the resids play an important role during reactions with coals. For example, the Blend resid and West Texas vacuum resids have a very similar elemental composition as well as similar carbon and hydrogen aromaticities but show different reactivity in the coprocessing reactions. At temperature of $450^{\circ} \mathrm{C}$ Blend resid is a poor coprocessing resid (liquid vehicle) while West Texas is relatively good. The combination of the analytical data indicates the structural differences of these two vacuum resids which probably in turn influences their reactivity as coprocessing solvents. West Texas vacuum resia is strongly aromatic, containing probably polyaromatic structures and ring substituents in the form of short alkyl groups. Although Blend vacuum resid has similar aromaticity, the way that the aromatic structures are connected with the aliphatic portion makes this resid perform so differently under coprocessing conditions. We propose here that the structure of the Blend vacuum resid consists of aromatic structures of smaller ring size connected with longer bridging groups. Hondo resid has a clearly strong paraffinic character which makes it a poor coprocessing feedstock. This information suggests that the different chemical structure of the petroleum resids define the resid as a 'good' or 'poor' coprocessing feedstock. 


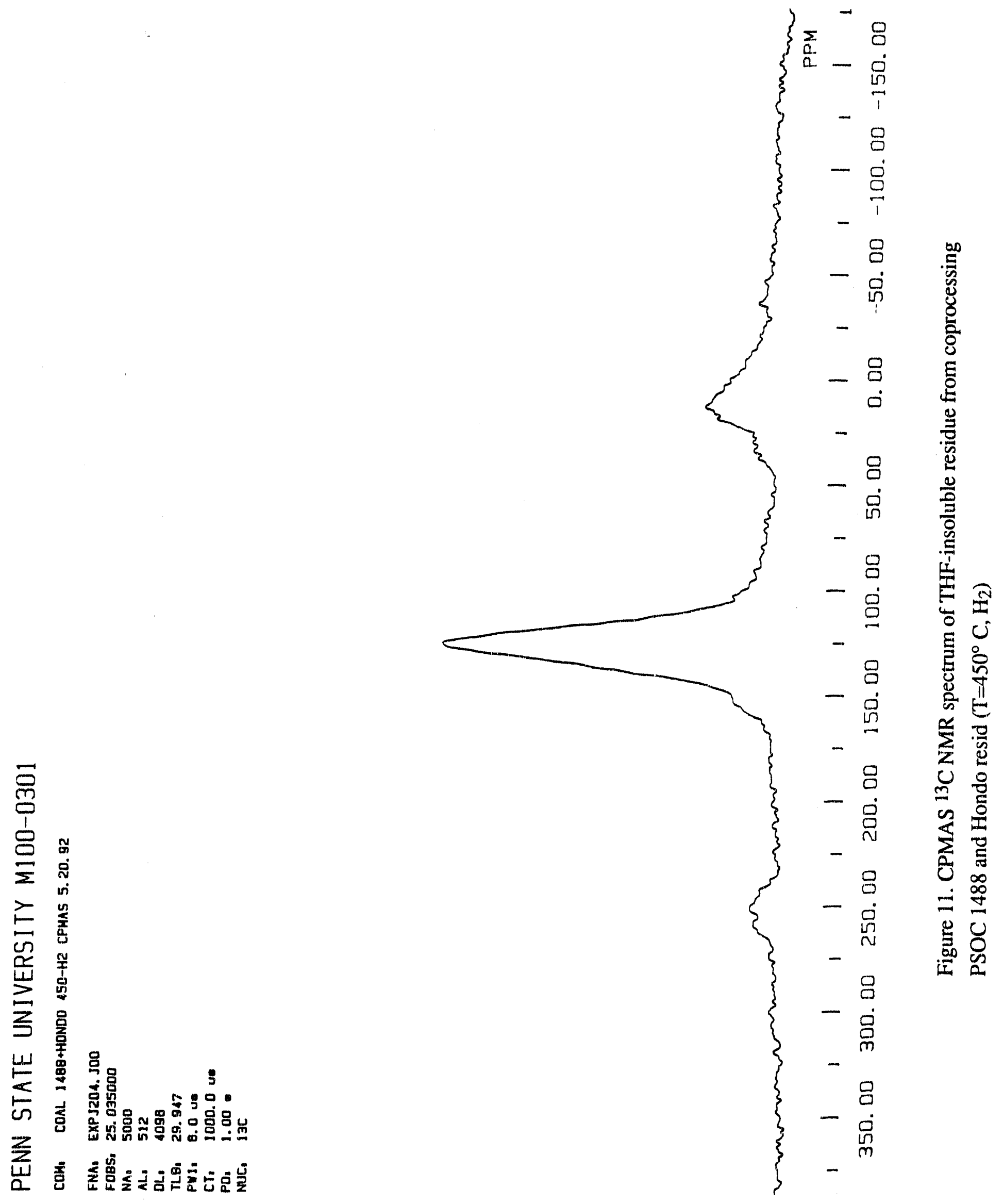




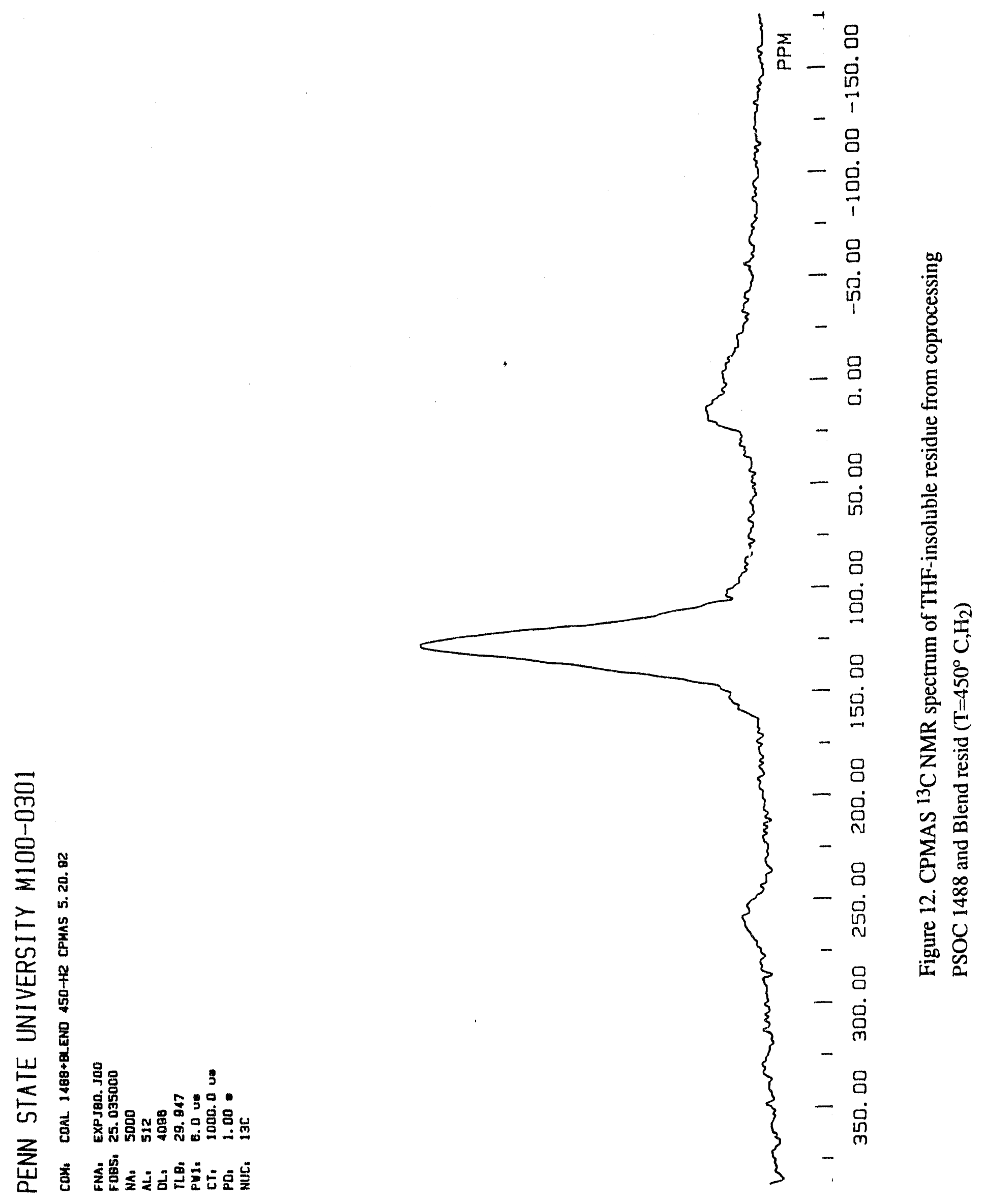




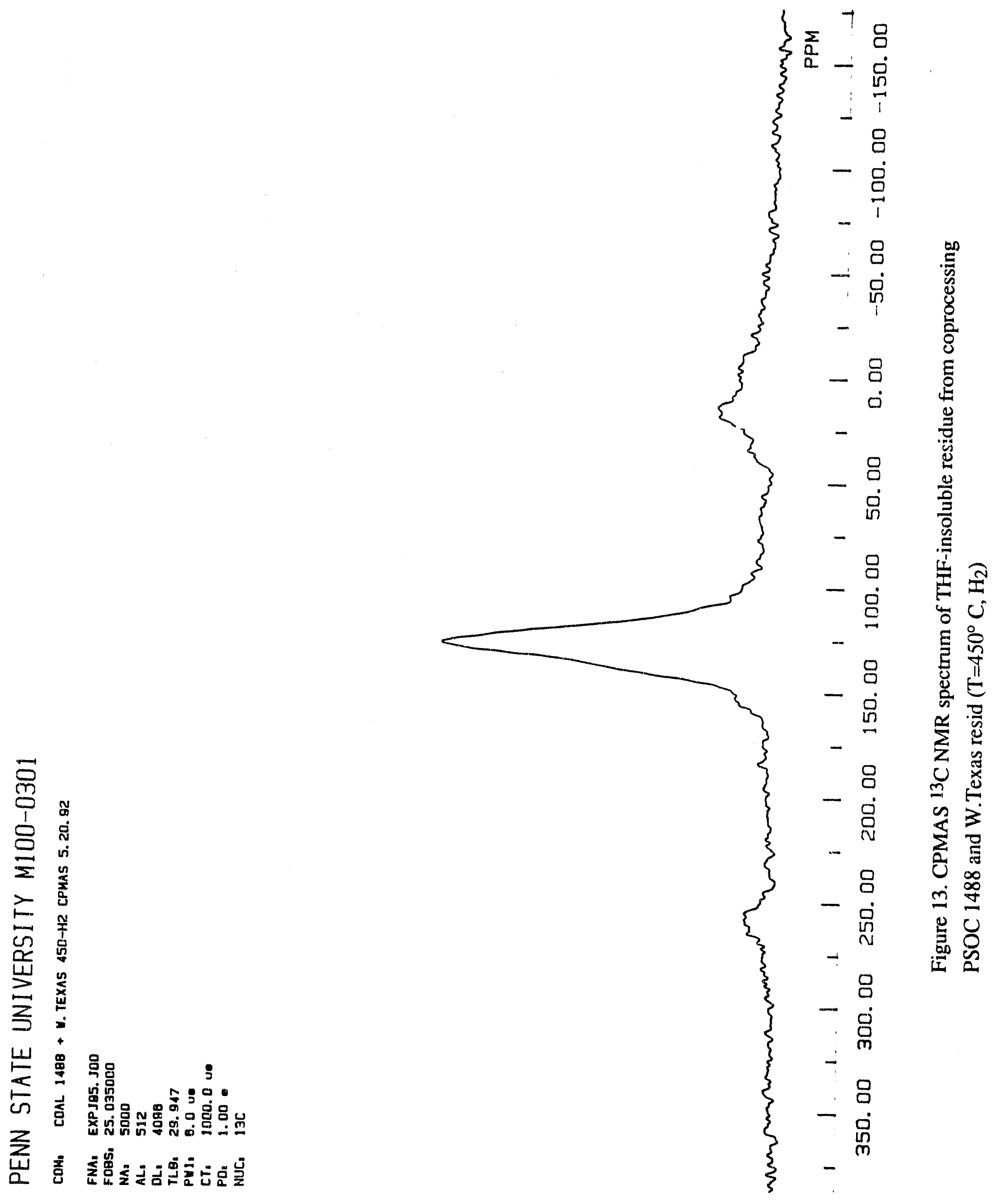




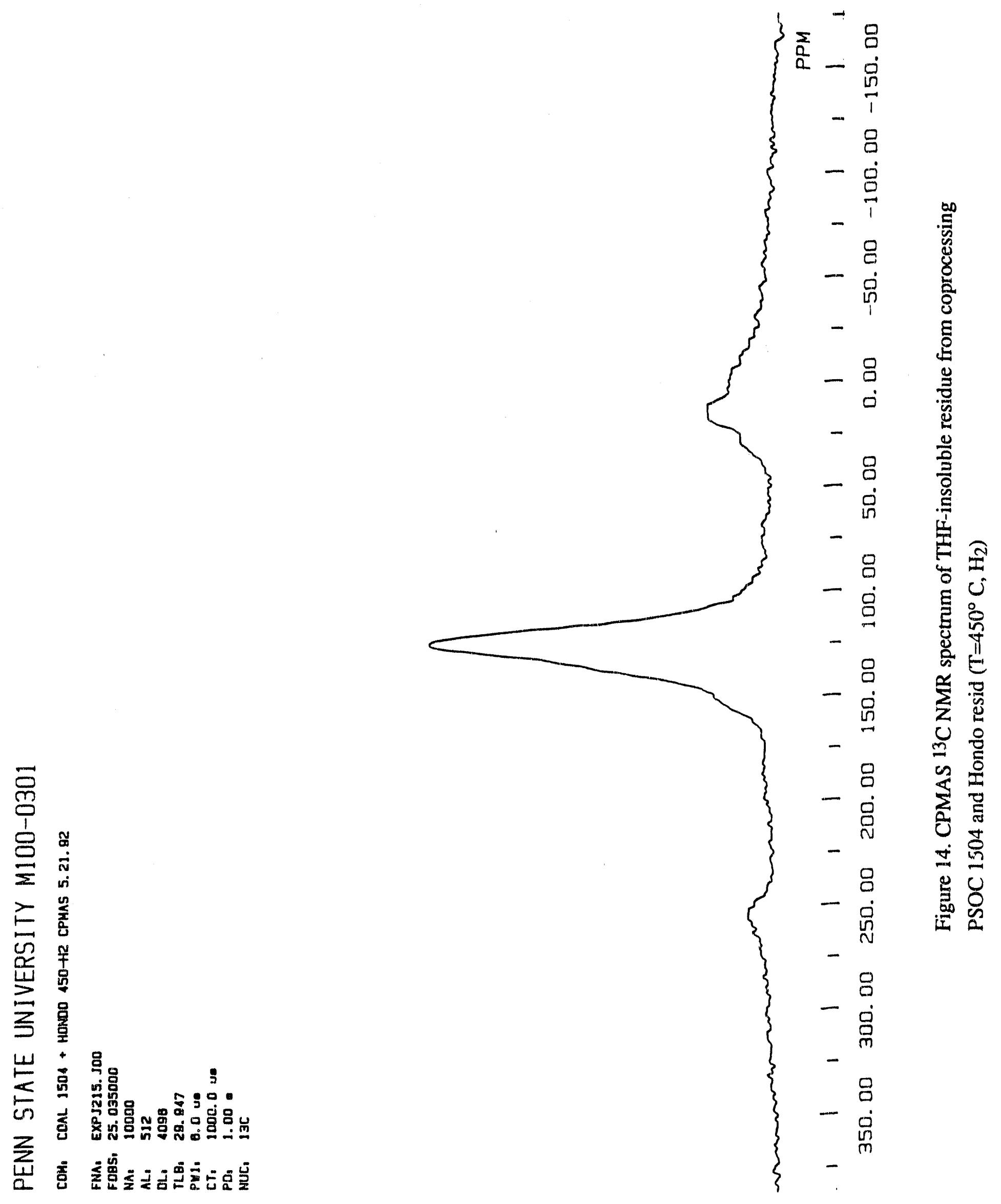




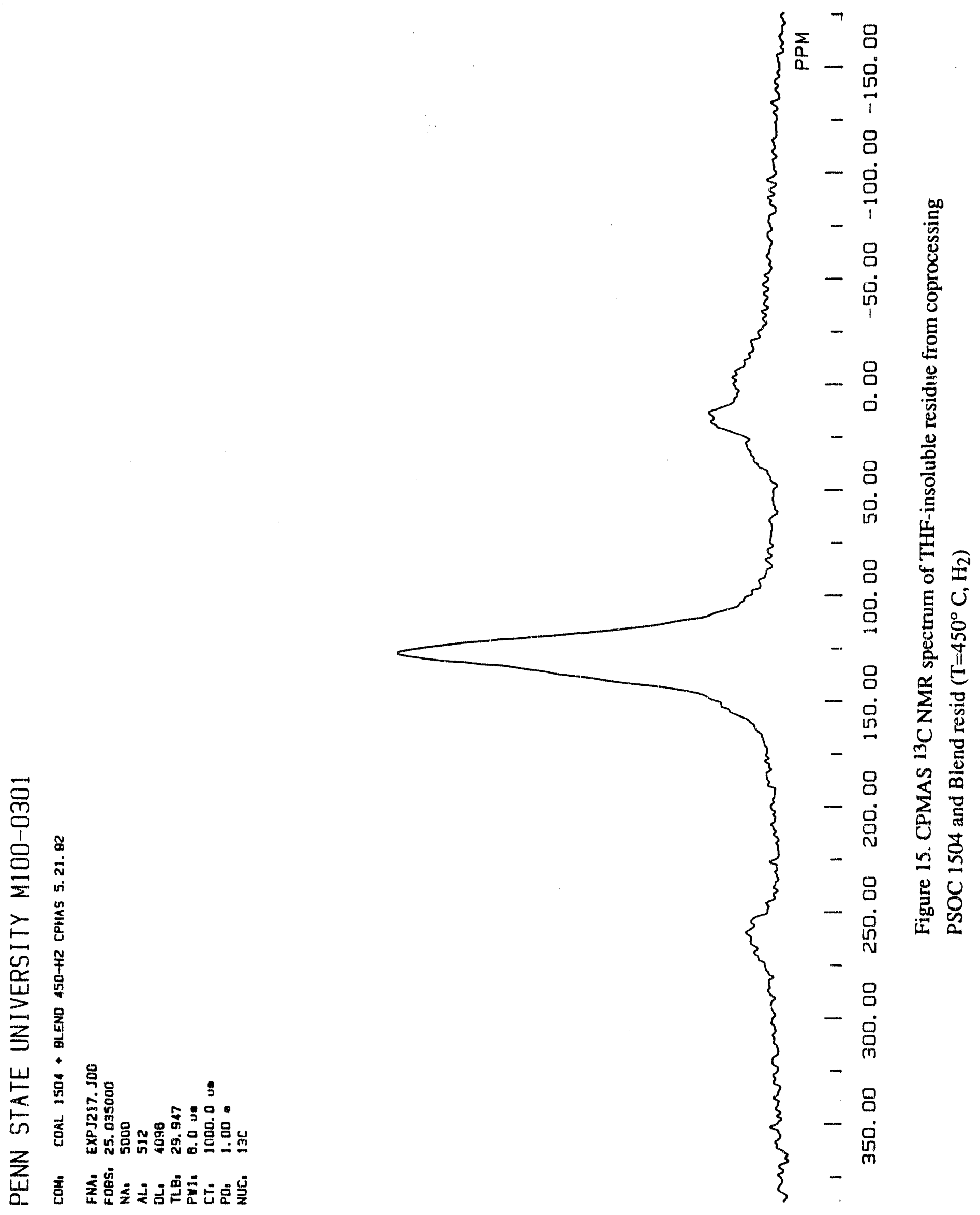




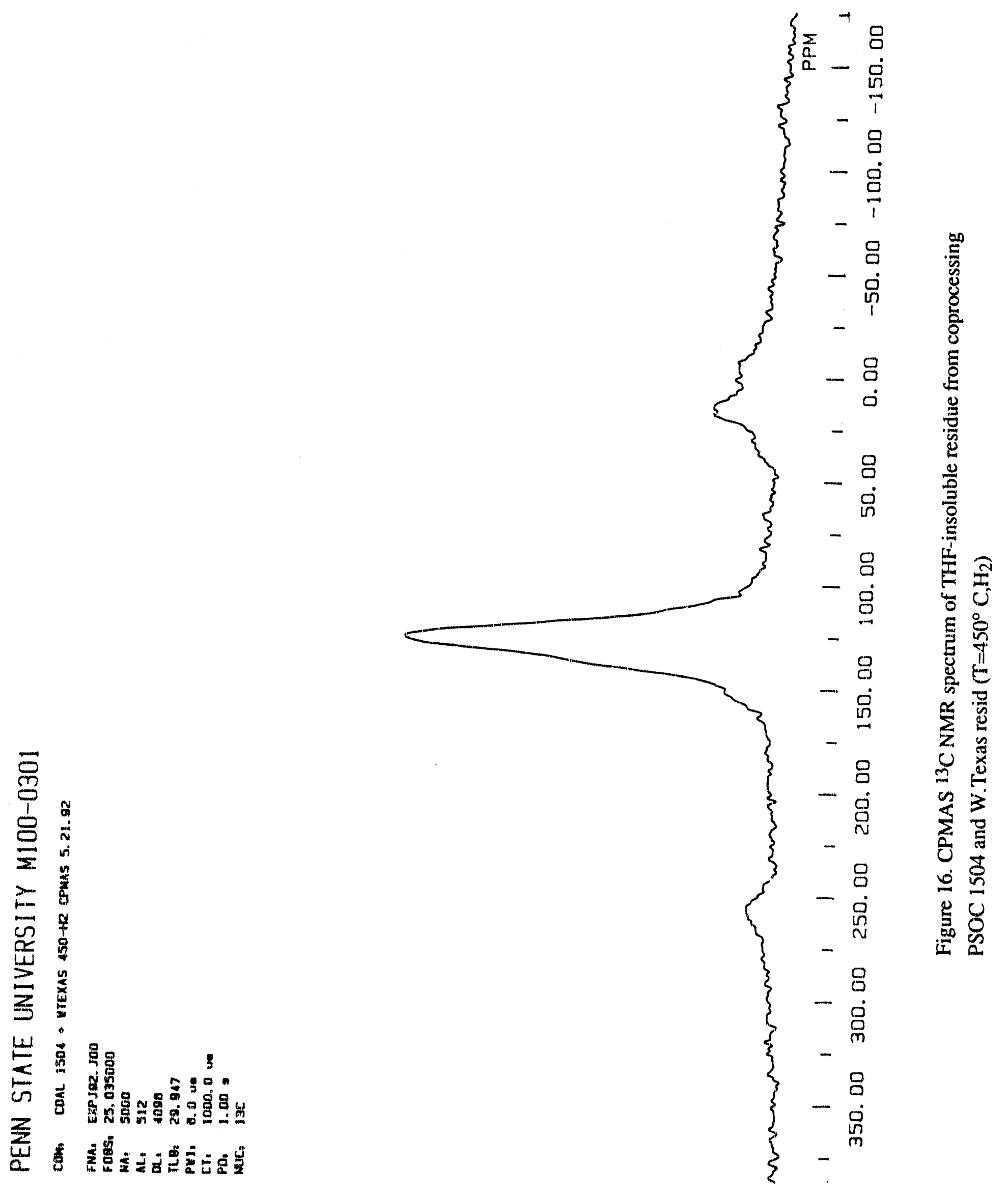




\section{LITERATURE CITED}

[1]. Schobert, H. H., J. Tomic, "Inhibition of Retrogressive Reactions in Coal/Petroleum Coprocessing", U.S.D.O.E. Report, No. 88PC-88935-QTR-12, 1991.

[2]. Schobert, H. H., J. Tomic, "Inhibition of Retrogressive Reactions in Coal/Petroleum Coprocessing", U.S.D.O.E. Report, No. 88PC-88935-QTR-13, 1991.

[3]. Schobert, H. H., J. Tomic, D. Moyer, and J. McConnie, "Inhibition of Retrogressive Reactions in Coal/Petroleum Coprocessing", U.S.D.O.E. Report, No. 88PC-88935-QTR-7, 1991.

[4]. Song, C.,S. Eser, H. H. Schobert, P. G. Hatcher, M. M. Coleman, and P. M. Walsh et. a 1.,"Advanced Thermally Stable Coal-Derived Jet Fuels", Technical Progress Report for Period July 1991 - November 1991, No. 18-1124-TPR-1 

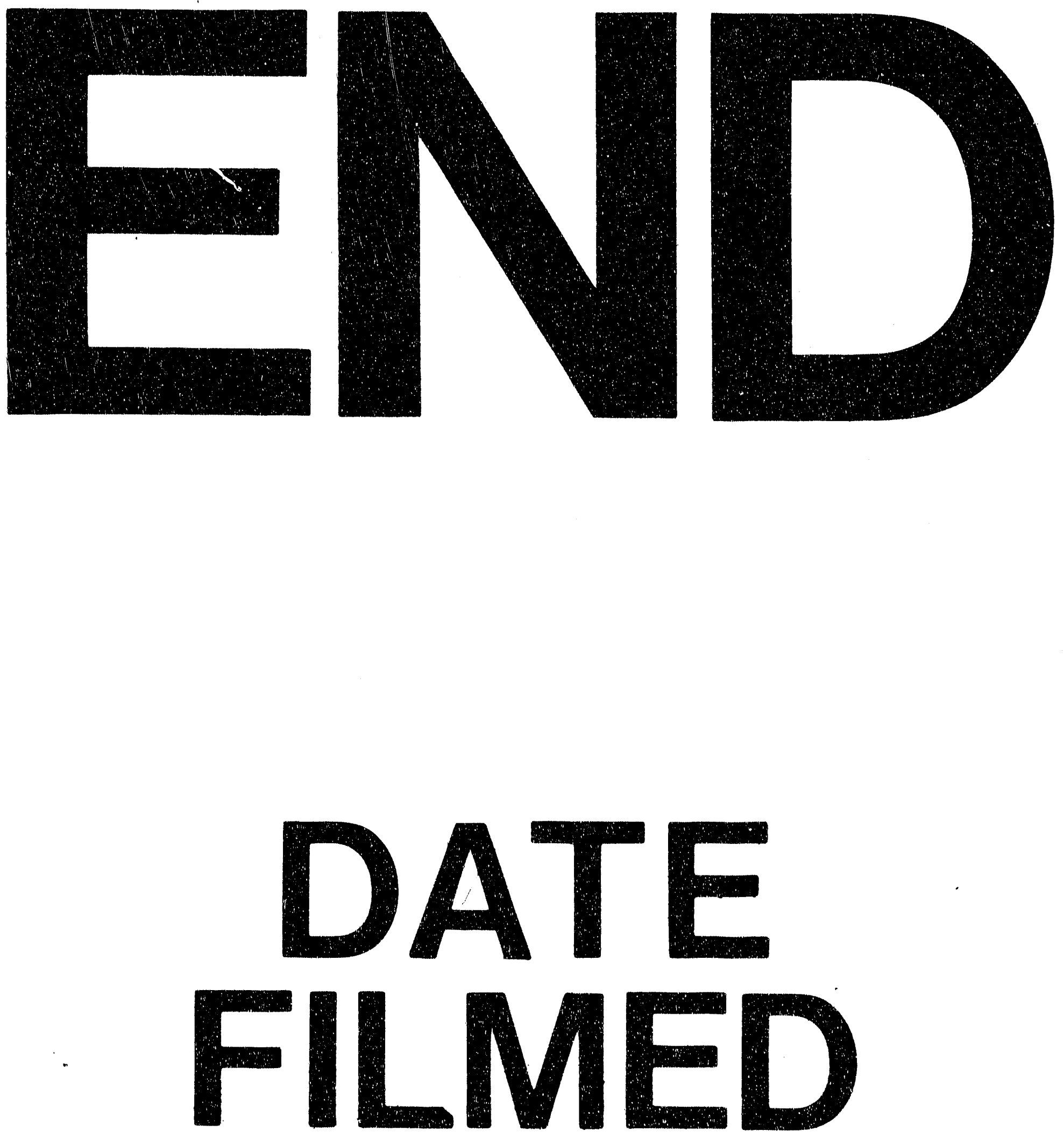

疌

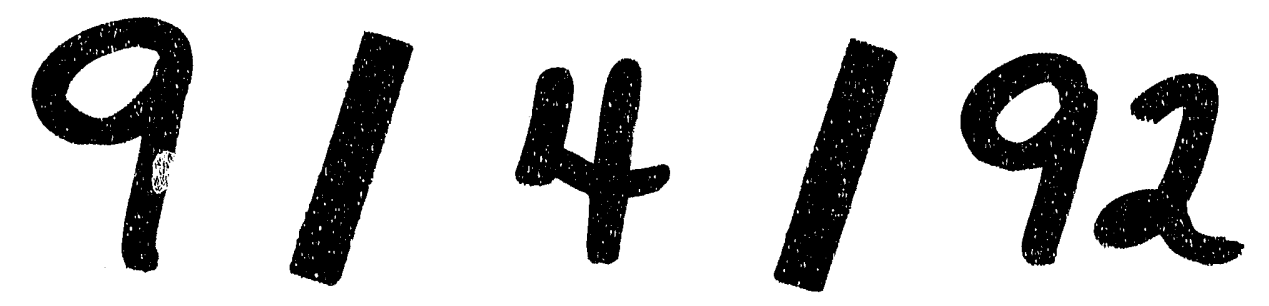


